\title{
Introducing radiality constraints in capacitated location-routing problems
}

\author{
Eliana Mirledy Toro Ocampo ${ }^{a^{*}}$, Frederico G. Guimarães ${ }^{b}$, Ramón Alfonso Gallego Rendónc
}

${ }^{a}$ Faculty of Industrial Enginnering, Technological University of Pereira. Pereira, Colombia

${ }^{b}$ Department of Electrical Engineering, Federal University of Minas Gerais, UFMG, Belo Horizonte, Brazil

${ }^{c}$ Electrical Engineering Program, Faculty of Engineering, Technological University of Pereira. Pereira, Colombia

CHRONICLE ABSTRACT

Article history:
Received October 272016
Received in Revised Format
December 252016
Accepted March 22017
Available online
March 32017
Keywords:
Capacitated vehicle routing
problem
Capacitated location-routing
problem
Combinatorial optimization
Radiality constraints
Spanning tree, vehicle routing
problem

In this paper, we introduce a unified mathematical formulation for the Capacitated Vehicle Routing Problem (CVRP) and for the Capacitated Location Routing Problem (CLRP), adopting radiality constraints in order to guarantee valid routes and eliminate subtours. This idea is inspired by formulations already employed in electric power distribution networks, which requires a radial topology in its operation. The results show that the proposed formulation greatly improves the convergence of the solver.

(C) 2017 Growing Science Ltd. All rights reserved

\section{Introduction}

Vehicle Routing Problems (VRP) is a general class of combinatorial optimization problems in operations research and transportation logistics. Much research has been done on theory, applications and methodologies for solving problems in this class (Dantzing \& Ramser, 1959; Magnati et al., 1995; Toth \& Vigo, 2002). VRP problems can be further divided into many other variants of problems, according to characteristics considered in the model and specificities required by the application domain.

In the Capacitated VRP (CVRP), there is an homogeneous fleet of vehicles $k=1, \ldots, K$ with limited capacity $C$ located in a single depot. There are $N$ clients with demands $D_{i}, i=1, \ldots, N$ that should be served. The cost of travelling between pairs of clients or between the depot and a client is represented by edges with associated costs. The goal is to find routes serving all clients with minimum cost. It is assumed that each route starts and ends in the depot, each client is visited only once and the capacity of each

* Corresponding author

E-mail: elianam@utp.edu.co (E. M. Toro Ocampo)

(C) 2017 Growing Science Ltd. All rights reserved. doi: $10.5267 /$ j.. jiec.2017.3.004 
vehicle should not be violated (Dantzing \& Ramser, 1959). The Capacitated Location-Routing Problem (CLRP) can be defined with a finite set of $N$ clients with demands $D_{i}, i=1, \ldots, N$ and a finite set of $M$ nodes representing potential depots, each one with an associated opening cost and determined capacity. There is an homogeneous fleet of vehicles $k=1, \ldots, K$ that should be routed from the depots to the clients. Each pair of nodes $i$ and $j$ has a cost $c_{i j}$. The goal is to determine the location of the depots and the routes from those depots to the clients with minimum cost. The total cost is given by the cost of opening depots and the costs of the routes. The CLRP is more complex because it considers three tasks simultaneously, namely locating, assigning and routing. The CVRP is a particular case of the CLRP, when only one depot is considered $(M=1)$.

The main purpose of this paper is to introduce new mathematical formulations for vehicle routing problems by using radiality constraints to eliminate subtours (Section 4). We present a structural similarity between valid solutions in optimization problems related to electrical power distribution systems and vehicle routing problems. The majority of electrical power distribution systems operate with radial topology for technical reasons related to coordination and protection. A radial configuration is one in which all nodes of the network are connected without loops, i.e. the configuration is a tree. Therefore optimization problems involving power distribution systems should have specific constraints to guarantee a minimum spanning tree, these are known as radiality constraints. The ideas conveyed here lead to new mathematical formulations for the CVRP and for the CLRP using radiality constraints. The contribution of this paper is to introduce new models to the literature that could be used and discussed by the OR community. The solution model guarantees a spanning tree becomes a subgraph formed only by Hamiltonian paths if each demand node (customer) has a degree less than equal to two. Whose characteristics ensure connected subgraphs, they prevent the appearance of solution proposals with subtours. The proposed model is compared with the model using the MTZ constraints (Miller et al., 1960) discussed in Section 3. The results clearly show that the new formulation greatly improves the convergence of the solver, see Section 5.

\section{Literature review}

The CVRP is characterized by a homogeneous fleet of vehicles with limited capacity, which is located at a single depot. There is a set of clients with known demand to be attended. The cost of travelling between nodes, which represent either clients or the single depot, is also known and is part of the input data of the problem. The goal is to find routes that serve all clients at minimum cost and visiting each client only once, respecting the capacity of the vehicles (Dantzing \& Ramser, 1959).

Christofides et al. (1981), consider three methods for the solution of the CVRP: Branch-and-Bound, savings algorithm (Clarke \& Wright, 1964) and the 3-opt local search. Jansen (1993), presents a two-step heuristic, route first, cluster second, based on the grand tour generation as proposed by (Christofides, 1969). Cornuejols and Harche (1993), propose a relaxation method for the CVRP by means of the Graphical VRP (GVRP).

Augerat et al. (1995), developed a Branch-and-cut algorithm, employing separation procedures. They also proposed a set of instances to validate the methodology. Ralphs et al. (2003), developed a parallel Branch, Cut and Price, with new branching rules and separation procedures. The methodology is validated with test instances taken from (Christofides et al., 1981; Augerat et al., 1995).

Gendreau et al. (2008), present an overview of metaheuristics applied to the CVRP: Simulated annealing, Tabu Search, Genetic Algorithms, Ant Colony Optimization, and Neural Networks. Prins (2004), implements a hybrid GA without route delimiters and employing local search operators. Chen et al. (2006), propose a hybrid algorithm combining Particle swarm optimization (PSO) and simulated annealing. Toth and Tramontani (2008), propose a local search algorithm based on the exploration of 
large scale neighborhoods. Letchford and Salazar-Gonzales (2015), present a survey of formulations for the CVRP based on variables commodity-flow and introduce new formulations called multi-commodity flow formulations. The proposed relaxations are strong regarding the determination of lower bounds. The authors have designed test instances up to 100 clients to demonstrate the efficiency of the method.

Regarding the CLRP, given its increased complexity, many heuristics have been developed to approach it within reasonable time. Nagy and Salhi (2007), classify these algorithms as sequential, iterative, hierarchical, or clustering based. Sequential methods initially decide the location of the depots and then define their respective routes. Salhi and Rand (1989), evaluate the effect of solving the location and routing problems separately. Iterative methods solve both subproblems simultaneously, feedbacking solutions obtained to each subproblem. Hierarchical methods consider the location problem as the main problem and the routing task as a subordinate problem. The location problem is solved with an approximation method, see for instance (Albareda-Sambola et al. 2005), (Melechovsky et al. 2005). Barreto et al. (2007), propose a methodology for the CLRP based on hierarchical clustering integrated in a sequential heuristic. To solve large scale instances (Prins et al., 2007), Prodhon (2011), Duhamel et al. (2010), Escobar et al. (2014), have developed many metaheuristics methods. Among these, Granular Tabu Search has been the most effective for instances up to 200 clients. Yu et al. (2009), proposed a Simulated annealing algorithm that found better gaps than the ones found in (Barreto et al., 2007) and (Prins et al., 2007). The location and routing problems are solved simultaneously. Hemmelmayr et al. (2012), developed an Adaptative large neighborhood search (ALNS) for the CLRP. In this technique different neighborhoods are considered and classified according to their performance. The most improving neighborhoods have their probabilities of use increased, thus being selected more often in the search.

The CLRP has been also solved by means of exact methods, including Branch and Price (Berger et al., 2007), column generation and Branch and Price (Akca et al., 2009), Branch and Cut (Belenguer et al. 2011). In Prodhon and Prins (2014), the authors present a complete study of LRP, analyzing and classifying different variants of the problem. The LRP has been solved with matheuristics techniques, combining heuristics and exact methods (Contardo et al., 2014), (Escobar et al., 2013), (Prins et al., 2007), (Ting \& Chen, 2013). These methods have been applied to instances with up 200 clients. However, some instances with 100 clients remain still unsolved.

\section{Mathematical model with classical MTZ constraints}

Let $I$ be the set of distribution centers (depots) and $J$ be the set of clients, such that the set of all vertices is $V=I \cup J$. Let $K$ be the set of vehicles. It is assumed a homogeneous fleet, such that all vehicles have the same capacity (Prins et al., 2007).

The variables used in the CLRP model are described next.

- $x_{i j k}$ : Binary variable indicating if the $\operatorname{arc}(i, j), i, j \in V$ is visited by vehicle $k \in K$.

- $y_{i}$ : Binary variable indicating if the depot $i \in I$ is opened.

- $f_{i j}$ : Binary variable indicating if client $j \in J$ is allocated to depot $i \in I$.

- $u_{i}$ : Number of cities visited when in the node $i \in V$.

- $t_{i j}$ : Real variable with the amount of cargo transported in $\operatorname{arc}(i, j), i, j \in V$.

The parameters of the model are: $O_{i}$ is the cost of opening the depot $i \in I ; W_{i}$ is the capacity of the depot $i \in I$; $Q$ is the capacity of the vehicle; $D_{j}$ is the demand of the customer located in node $j \in J$; $c_{i j}$ is the cost of travelling from node $i$ to node $j ; F$ is the fixed cost associated with each vehicle utilized in the operation. 
The mathematical model with the classical MTZ constraints is presented next.

$$
\begin{aligned}
& \min \sum_{i \in I} O_{i} y_{i}+\sum_{i \in V} \sum_{j \in V} \sum_{k \in K} c_{i j} x_{i j k}+\sum_{i \in I} \sum_{j \in J} \sum_{k \in K} F x_{i j k} \\
& \sum_{i \in V} \sum_{k \in K} x_{i j k}=1 \\
& \forall j \in J \\
& \sum_{i \in V} \sum_{j \in J} D_{j} x_{i j k} \leq Q \\
& \forall k \in K \\
& \sum_{j \in V} x_{i j k}-\sum_{j \in V} x_{j i k}=0 \\
& \forall i \in V, \forall k \in K \\
& \sum_{i \in I} \sum_{j \in J} x_{i j k} \leq 1 \\
& \forall k \in K \\
& \sum_{u \in J} x_{i u k}+\sum_{u \in V, u \neq j} x_{u j k} \leq 1+f_{i j} \\
& \forall i \in I, \forall j \in J, \forall k \in K \\
& \sum_{j \in J} D_{j} f_{i j} \leq W_{i} y_{i} \\
& \forall i \in I \\
& u_{i}-u_{j}+|J| x_{i j k} \leq|V|-|I| \\
& \sum_{j \in J} t_{i j} \leq W_{i} y_{i} \\
& \sum_{j \in J} t_{i j} \leq Q|K| y_{i} \\
& \sum_{k \in K} Q x_{i j k} \geq t_{i j} \\
& \sum_{h \in V, h \neq i} t_{h i}=\sum_{j \in V, j \neq i} t_{i j}+D_{i} \\
& \sum_{i \in I} f_{i j}=1 \\
& x_{i j k}+x_{j i k}=1 \\
& \sum_{i \in I} y_{i} \geq\left(\frac{1}{\sum_{i \in I} W_{i}}\right) \sum_{j \in J} D_{j} \\
& \sum_{i \in I} \sum_{j \in J} \sum_{k \in K} x_{i j k} \geq \sum_{j \in J} D_{j} / Q
\end{aligned}
$$

Additionally, we have $x_{i j k} \in\{0,1\}, y_{i} \in\{0,1\}, f_{i j} \in\{0,1\}, u_{i} \geq 0$, and $t_{i j} \geq 0$.

The objective function (1) sums the costs of those depots opened, the costs of the visited arcs in the routes and the fixed costs associated with the use of the vehicles. It represents the simultaneous minimization of the costs of opening depots, the costs of the arcs selected in the definition of the routes and the number of vehicles used in the distribution network. Constraints (2) guarantee that all clients are visited. Constraints (3) and (7) are related to the capacities of the vehicles and depots respectively. Constraints (4) and (5) ensure the continuity of the route such that each route starts and ends at the same depot. Constraints (6) specify that a client can be assigned to a depot only if there is a route for that depot. The elimination of subtours using the classical MTZ constraints are expressed in (8). Constraints (9)-(12) basically allow defining the route (topology and flows). 
Constraints (9) and (10) limit the sum of the flows in the arcs emerging from a depot (depending on whether the warehouse is built, according to the respective variable $y_{i}$. Constraint (11) limits the flow in an arc in terms of the variables $x$ that define the state of the vehicle in that arc. Constraint (12) represents the load balance on a node related to a customer.

Constraints (13) indicate that customer demand must be connected to the depot. Constraints (14) indicate that any arc is active in only one route. Constraints (15) determine an inferior limit to the number of depots used according to the sum of the demand and the capacities of the depots. Constraints (16) guarantee that the number of routes is sufficient to supply the demand.

The model presented by (Prins et al., 2007) was increased with the constraints (9) to (16), in order to place it on equal terms with the model presented in section 4 of this article. This model can be adapted to different variants of the VRP. For instance, in order to have a CVRP, one can specify the set of depots with only one node. Objective function (1) should change accordingly, for the CVRP only considers the costs of the active arcs.

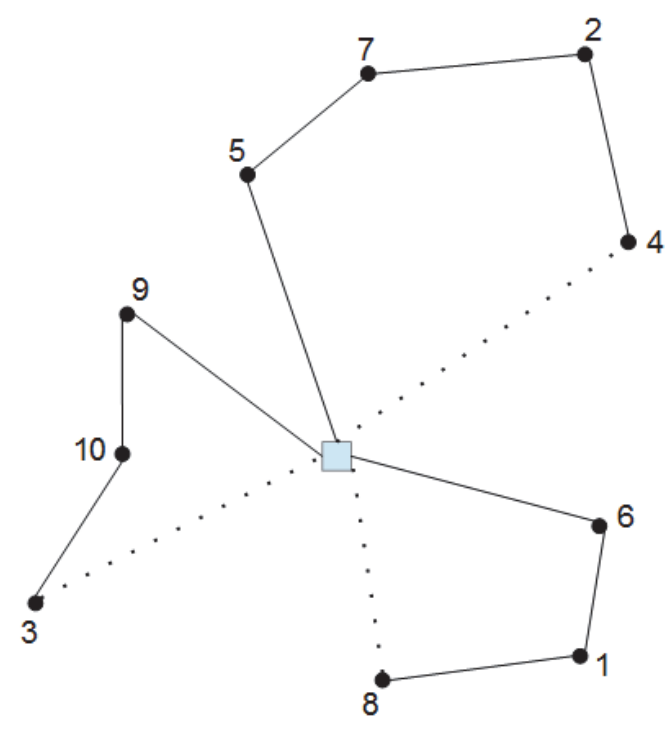

Fig. 1. Typical topology of a VRP solution.

\section{CLRP model proposed with radial constraints}

Optimization problems in power distribution systems involve changing the topology of the network in order to improve its characteristics, such as reducing power losses, balancing loads, and improving voltage profile in the network (Lavorato et al., 2012). These problems assume the availability of controllable switches (manual or automatic) throughout the network that can be used to change the topology of the distribution system network (Prado et al., 2014). The majority of electrical power distribution systems operate with radial topology for technical reasons related to coordination and protection. A radial configuration is one in which all nodes of the network are connected without loops, i.e. the configuration is a tree.

Fig. 1 illustrates a possible solution to the VRP involving one depot. The dashed line represents the edge between the last client in the route and the depot. For a valid solution to the VRP, if we neglect the return arc, the topology of this solution is a tree, i.e. the topology becomes radial. There is a direct association between depots in VRP and substations in power distribution problems. Each substation has an associated tree connecting the supply to the demand nodes. Similarly, each depot has an associated tree representing 
the (open) routes connecting clients to the depot. Following this idea, radiality constraints could be in principle used to eliminate subtours in the VRP in much the same way they are used to eliminate loops in valid power distribution networks. Therefore, the main idea and motivation in the paper is to embed the radiality constraints in the model of the VRP.

A discussion on radial constraint formulations, in which the radial configuration is established as a subgraph, can be found in papers related to the optimization of planning and operation of power distribution systems, see for instance (Lavorato et al., 2012). In this problem, a configuration implies that there is only one way between the substation of power energy supply and the end users, the continuity of service being a priority for the power utility companies. One way to ensure the continuity of service in the network is dealing with the reconfiguration problem, in which the states of normally open or normally closed switches are changed to modify the topology of the network. The necessary condition to represent the radial characteristics in the vehicle routing problem requires that the number of arcs be equal to the number of client nodes minus one, in the case of having only one depot. When there are $m$ depots, the necessary condition becomes the number of arcs should be equal to the number of client nodes minus the number of depots.

The radiality constraint is then guaranteed by constraint (23). This constraint is necessary but not sufficient. This constraint is complemented with other constraints, (18) and (19), the first one imposes that each client node has an arc connecting it to the route and the second one guarantees that for each client node, the number of arcs leaving the node should be equal to the number of arcs entering the node, except for a terminal node.

This model can be adapted to open routing problems, closed routing problems and combinations of these. In the case of open vehicle routing problems, there are nodes which are terminal nodes, that is, the vehicle does not need to return to the depot after attending to this client. The client node identified as terminal is the last delivery of the vehicle in that route. In the case of closed vehicle routing problems, the vehicles should return to its corresponding depot when reaching to the terminal node, which is enforced by constraints (27)-(30), by identifying the arc that connects the node at the end of the route with the depot, as determined by $a_{i j}$ in the mathematical model. Finally, the third case considers a combination of open and closed routes. This is much more complex from the mathematical point of view. The model proposed in this paper covers the second case, the one with closed routes.

In addition to the necessary constraints, there exist the sufficient ones which establish the feasibility of the configurations. In power systems, a disconnected network produces an infeasible solution, due to the interruption of service in the delivery of energy to the non-connected customers. To solve this situation, in the context of power systems, there are constraints to ensure the connectivity; those are the power balance equations imposed on each node of the network.

Similar to the context of power distribution problems, in the vehicle routing problem the connectivity constraints are obtained by means of the load balance in the points of connection. Therefore, constraint (22) guarantees the connectivity of the network through the balance of demands in the client nodes. This constraint not only guarantees the connectivity but also avoids the formation of disconnected regions.

The variables used in the proposed CLRP model are described next.

- $x_{i j}$ : Binary variable indicating if the arc $(i, j), i, j \in V$, is used

- $y_{i}$ : Binary variable indicating if the depot $i \in I$ is opened.

- $z_{j}$ : Binary variable indicating if the client $j \in J$ is the last one in the route.

- $f_{i j}$ : Binary variable indicating if client $j \in J$ is allocated to depot $i \in I$. 
- $a_{i j}$ : Binary variable indicating if the vehicle is returning from node $j \in J$ to the depot $i \in I$

- $t_{i j}$ : Real variable with the amount of cargo transported in arc $(i, j), i, j \in V$

The parameters of the model are the same of the previous model. The proposed model is introduced below:

$$
\begin{aligned}
& \min \sum_{i \in I} O_{i} y_{i}+\sum_{i \in V} \sum_{j \in V} c_{i j} x_{i j}+\sum_{i \in I} \sum_{j \in J} c_{i j} a_{i j}+\sum_{i \in I} \sum_{j \in J} F a_{i j} \\
& \sum_{i \in V} x_{i j}=1 \\
& \sum_{k \in J} x_{j k}+\sum_{i \in I} a_{i j}=\sum_{i \in V} x_{i j} \\
& \forall j \in J \\
& \forall j \in J \\
& \sum_{j \in J} x_{i j}=\sum_{j \in J} a_{i j} \\
& \forall i \in I \\
& x_{i j}+x_{j i} \leq 1 \\
& \sum_{i \in V, i \neq j} t_{i j}=\sum_{k \in V, k \neq j} t_{j k}+D_{j} \\
& \forall i, j \in V \\
& \forall j \in J \\
& \sum_{i \in V} \sum_{j \in V} x_{i j}=|J| \\
& \sum_{i \in I} f_{i j} \leq 1 \\
& \forall j \in J \\
& t_{i j} \leq Q x_{i j} \\
& \sum_{j \in J} t_{i j} \leq W_{i} y_{i} \\
& \forall i, j \in V \\
& \forall i \in I \\
& \sum_{k \in V} x_{j k}=1-z_{j} \\
& \forall j \in J \\
& 1+a_{i j} \geq f_{i j}+z_{j} \\
& \forall i \in I, \forall j \in J \\
& -\left(1-x_{j u}-x_{u j}\right) \leq f_{i j}-f_{i u} \\
& \forall i \in I, \forall j, u \in V \\
& f_{i j}-f_{i u} \leq\left(1-x_{j u}-x_{u j}\right) \\
& \forall i \in I, \forall j, u \in V \\
& f_{i j} \geq x_{i j} \\
& \forall i \in I, \forall j \in J \\
& \sum_{i \in I} y_{i} \geq\left(\frac{1}{\sum_{i \in I} W_{i}}\right) \sum_{j \in J} D_{j} \\
& \sum_{i \in I} \sum_{j \in J} x_{i j} \geq \sum_{j \in J} D_{j} / Q \\
& \sum_{i \in I} \sum_{j \in J} a_{i j} \leq|K|
\end{aligned}
$$

Additionally, we have $x_{i j} \in\{0,1\}, y_{i} \in\{0,1\}, f_{i j} \in\{0,1\}, z_{j} \in\{0,1\}, a_{i j} \in\{0,1\}$, and $t_{i j} \geq 0$

The objective function (17) considers the operational costs, which correspond to the costs of opening depots, costs of opening routes, and costs associated to the edges used in the routes.

All demand nodes $j \in J$ should have an arriving arc connecting it to the route (18). Constraints (19) guarantee the number of arcs leaving the node should be equal to the number of arcs arriving at the node. 
Constraints (20) guarantee that for a depot $i \in I$ there is one arc leaving the depot and one arc returning to it. Constraints (21) avoid duplication of arcs, defines the orientation of an arc, if it is from $i$ to $j$ or vice-versa.

Constraints (22) represent the flow balance of the routes. Constraints (23) identify the active arcs to generate the radial topologies. Constraints (24) guarantee that the demand of a route is connected to a distribution center. Constraints (25) limit the flow in a route according to the capacity of the vehicles.

Constraints (26) limit the flow leaving one depot according to its capacity and the decision to use this node as a distribution center. Constraints (27) define a terminal node for the routes when there is no arc leaving that demand node. If $j$ is a terminal node, then constraints (28) guarantee that there is a return arc. Constraints (29) and (30) identify the active arcs and the starting node of the route in order to activate the return arc to the depot. If the arc between depot $i$ and the node $j$ is active, then node $j$ is connected to the depot $i\left(f_{i j}=1\right)$, as expressed in (31). Constraints (32) determine an inferior limit to the number of depots used according to the sum of the demand and the capacities of the depots. Constraints (33) guarantee that the number of routes is sufficient to supply the demand. Constraints (34) establish the maximum number of routes attended by the fleet of vehicles available.

Similarly to the model with MTZ constraints, the one proposed with radial constraints is flexible and can be adapted to other variants of the VRP, including the CVRP.

It is important to emphasize that constraints (22) and (23) guarantee feasible solutions and avoid the appearance of solutions with sub tours, and ensure that all customers are connected through radial topology (a minimum spanning tree). These constraints are complemented by (18) and (19) this indicates that the grade of each demand node must be less than equal or equal to 2 . So each feasible solution obtained consists only Hamiltonian paths. The interesting aspect of the radial model for vehicle routing is that all valid configurations connect customers to the depots by means of trees, therefore ensuring the elimination of cycles. This different perspective about the problem is the basis of the radial model proposed in this paper.

\section{Results}

The computational experiments were done in a computer Core i7-4770 $3.4 \mathrm{GHz}, 16 \mathrm{~GB}$ of RAM. The mathematical model was solved with CPLEX 12.5 (ILOG, 2008) and written in AMPL (Fourer et al., 2002). In order to compare the performance of the mathematical models, we adopted the instances of the CVRP available in (Augerat et al., 1995) and the instances of the CLRP available in (Duhamel et al., 2010). For the CVRP, we consider set $P$ proposed (Augerat et al. 1995), where the number of customers $\mathrm{N}$ is between 15 and 100, and the capacity of vehicle (C) is between 35 and 3000 . The data set for the CLRP contains 30 instances. The number of depots is either 5 or 10 , the number of customers $\mathrm{N}$ is in the set $\{20,50,100,200\}$ and the capacity $\mathrm{C}$ is in the set $\{70,150\}$. Note that, as done in previous studies on CLRP, the weight of the travel distance is set to be 100, which means that the travel distances are multiplied by 100 for all instances in this dataset.

First, we apply the mathematical model with MTZ constraints and the proposed model with radiality constraints to the set of instances of the CVRP. The results are presented in Table 1. In this table, the GAP is reported in two ways:

- the difference between the upper bound and the lower bound in CPLEX

$$
G A P B=\frac{U B-L B}{U B} \times 100 \%
$$


- the difference between the best known solution and the best solution in CPLEX

$$
G A P_{B K S}=\frac{B K S-U B}{B K S} \times 100 \%
$$

Table 1

Results for the instances of the CVRP

\begin{tabular}{lcccccccccc}
\hline \multicolumn{1}{c}{ Instances } & \multicolumn{1}{c}{ Radial Model } \\
\hline Instance & $K$ & BKS & $\begin{array}{c}G A P_{B} \\
(\%)\end{array}$ & $\begin{array}{c}G A P_{B K S} \\
(\%)\end{array}$ & $\begin{array}{c}\text { No. } \\
\text { branches }\end{array}$ & $\begin{array}{c}\text { Time } \\
(s)\end{array}$ & $\begin{array}{c}G A P_{B} \\
(\%)\end{array}$ & $\begin{array}{c}G A P_{B K S} \\
(\%)\end{array}$ & $\begin{array}{c}\text { No. } \\
\text { branches }\end{array}$ & $\begin{array}{c}\text { Time } \\
(s)\end{array}$ \\
\hline P-n19-k2 & 2 & 212 & 1.94 & 0.00 & 4003 & 2 & 5.79 & 0.00 & 3202 & 2 \\
P-n20-k2 & 2 & 216 & 2.86 & 0.00 & 1736 & 1 & 5.02 & 0.46 & 986 & 1 \\
P-n21-k2 & 2 & 211 & 3.04 & 0.00 & 518 & 1 & 3.04 & 0.00 & 518 & 1 \\
P-n22-k2 & 2 & 216 & 1.73 & 0.00 & 1095 & 1 & 14.88 & 12.96 & 17 & 1 \\
P-n22-k8 & 8 & 603 & 0.50 & 0.00 & 20275 & 31 & 4.80 & 0.16 & 1462 & 31 \\
P-n23-k8 & 8 & 529 & 3.36 & 0.00 & 57913 & 105 & 6.08 & 0.56 & 3366 & 105 \\
P-n40-k5 & 5 & 458 & 0.31 & 0.00 & 36187 & 150 & 7.74 & 3.71 & 1775 & 150 \\
P-n45-k5 & 5 & 510 & 1.15 & 0.00 & 283463 & 1920 & 8.85 & 5.29 & 18730 & 1920 \\
P-n50-k7 & 7 & 554 & 1.69 & 0.00 & 270948 & 3600 & 10.45 & 6.50 & 7454 & 3600 \\
P-n65-k10 & 10 & 792 & 4.10 & 0.75 & 627504 & 35000 & 27.25 & 28.79 & 8831 & 35000 \\
P-n76-k4 & 4 & 593 & 0.00 & 0.00 & 456515 & 8853 & 18.35 & 19.89 & 35837 & 18500 \\
P-n76-k5 & 5 & 627 & 2.64 & 0.32 & 495833 & 30000 & 24.71 & 27.91 & 37100 & 30000 \\
P-n101-k4 & 4 & 681 & 0.25 & 0.00 & 349788 & 10000 & 42.14 & 42.14 & 4048 & 10000 \\
\hline
\end{tabular}

Table 2

Results for the instances of the CLRP.

\begin{tabular}{|c|c|c|c|c|c|c|c|c|c|c|c|}
\hline \multicolumn{4}{|c|}{ Instances } & \multicolumn{4}{|c|}{ Radial Model } & \multicolumn{4}{|c|}{ MTZ Model } \\
\hline Instance & BKS & $K$ & $M$ & $\begin{array}{c}G A P_{B} \\
(\%)\end{array}$ & $\begin{array}{c}G A P_{B K S} \\
(\%)\end{array}$ & $\begin{array}{c}\text { No. } \\
\text { branches }\end{array}$ & $\begin{array}{c}\text { Time } \\
(s)\end{array}$ & $\begin{array}{c}G A P_{B} \\
(\%)\end{array}$ & $\begin{array}{c}G A P_{B K S} \\
(\%)\end{array}$ & $\begin{array}{c}\text { No. } \\
\text { branches }\end{array}$ & Time $(s)$ \\
\hline $20-5-1 a$ & 54793 & 5 & 3 & 1.08 & 0.00 & 166428 & 580 & 5.32 & 0.42 & 14326 & 580 \\
\hline $20-5-1 b$ & 39104 & 3 & 2 & 2.48 & 0.00 & 627 & 8 & 24.54 & 26.95 & 5 & 8 \\
\hline $20-5-2 a$ & 48908 & 5 & 2 & 0.00 & 0.00 & 109034 & 210 & 25.90 & 13.46 & 3599 & 210 \\
\hline $20-5-2 b$ & 37542 & 3 & 2 & 0.00 & 0.00 & 2600 & 3 & 20.03 & 20.34 & 5 & 3 \\
\hline $50-5-1 a$ & 90111 & 12 & 3 & 6.65 & 0.05 & 295084 & 18000 & 45.20 & 50.45 & 19640 & 18000 \\
\hline 50-5-2bbis & 51822 & 6 & 3 & 0.62 & 0.00 & 973765 & 16000 & 13.55 & 7.14 & 12946 & 18000 \\
\hline $100-5-1 \mathrm{a}$ & 275457 & 25 & 3 & 7.10 & 3.44 & 139430 & 85000 & $\mathrm{~N} / \mathrm{A}$ & N/A & & \\
\hline
\end{tabular}

In the instances considered, it is possible to observe that the radial model is able to obtain the BKS in all cases, except for instances P-n65-k10 and P-n76-k5, with a gap of 0.75 and $0.32 \%$ respectively. The MTZ model is able to achieve the BKS only for two small instances with 19 and 21 clients. In the other instances, the difference with respect to the BKS is from $0.33 \%$ to $42.12 \%$. In all instances, the same time limit is considered. With respect to the difference between upper and lower bounds, the radial model achieves GAP between $0.00 \%$ and 5.08\%. The MTZ model achieves GAP between $4.63 \%$ and 31.09\%.

In the CLRP, we considered instances from 20 to 100 clients and 5 depots. The radial model is able to obtain the BKS in the majority of instances, except instance $100-5-1 \mathrm{a}$, presenting a GAP of $3.44 \%$. The MTZ model does not achieve the BKS in any of the instances, presenting GAP between $0.44 \%$ and $50.45 \%$. In instance 100-5-1a, it does not even achieve any gap. Regarding the difference between the upper and lower bounds, the radial model achieves GAP ranging between $0.00 \%$ and $7.10 \%$, while the MTZ model achieves GAP ranging between $5.32 \%$ and $45.20 \%$ and no gap is achieved for some instances. 
The computational time limit was the same for all instances of the CVRP and the CLRP. One can notice that the number of branches analyzed when using the radial model is much higher, showing that the radial model allows a better exploration of the search tree than the MTZ model.

\section{Conclusion}

In this paper, new formulations for the CVRP and the CLRP were presented in order to minimize operational costs and investment costs. The main motivation was to develop new formulations able to achieve the optimal solutions faster. The new formulations were based on the radiality constraints already used in MILP formulations of power distributions network problems, in which the operation assumes a radial topology for the network. These radiality constraints are used to guarantee the radial topology (a minimum spanning tree) and eliminate loops in the network, and ensure every feasible solution consists only Hamiltonian paths. By identifying the similarity between this topology and valid solutions for VRP, we introduce a new formulation for the CVRP and the CLRP.

The computational experiments revealed that the formulation with radiality constraints presented best performance for all the instances. When the problem size grows, the performance of radiality constraints improves compared with the model with MTZ. It was noticed that the radiality constraints formulation was able to achieve the optimum in more instances and in those cases when no convergence to the optimum was achieved, at least better gaps were produced in most cases. Optimality has been achieved in many instances of the CVRP and the CLRP. It was also observed that the radial model presents a better capacity of exploration of the search tree, considering the same computing times.

In future work, we intend to apply these formulations to other variants of VRP problems in the literature, such as the Multi-depot VRP, Open VRP, CLORP and Multi-Depot Open VRP, given the radial nature of these problems. Additionally, we intend to employ the faster models as local search procedures within metaheuristics for the large scale instances in a hybrid scheme.

\section{Acknowledgments}

E. M. Toro would like to thank the support given by Faculty of Industrial Engineering and the Vicechancellorship for Research, innovation and outreach of the Technological University of Pereira, Colombia. F. G. Guimarães would like to thank the National Council for Scientific and Technological Development (CNPq grant no. 312276/2013-3), Brazil.

\section{References}

Albareda-Sambola, M., Díaz, J. A., \& Fernández, E. (2005). A compact model and tight bounds for a combined location-routing problem. Computers \& Operations Research, 32(3), 407-428.

Akca, Z., Berger, R. T., \& Ralphs, T. K. (2009). A branch-and-price algorithm for combined location and routing problems under capacity restrictions. In Operations research and cyberinfrastructure (pp. 309-330). Springer US.

Augerat, P., Belenguer, J. M., Benavent, E., Corberán, A., Naddef, D., \& Rinaldi, G. (1998). Computational results with a branch-and-cut code for the capacitated vehicle routing problem.

Barreto, S., Ferreira, C., Paixao, J., \& Santos, B. S. (2007). Using clustering analysis in a capacitated location-routing problem. European Journal of Operational Research, 179(3), 968-977.

Belenguer, J. M., Benavent, E., Prins, C., Prodhon, C., \& Calvo, R. W. (2011). A branch-and-cut method for the capacitated location-routing problem. Computers \& Operations Research, 38(6), 931-941.

Berger, R. T., Coullard, C. R., \& Daskin, M. S. (2007). Location-routing problems with distance constraints. Transportation Science, 41(1), 29-43. 
Christofides, N., Mingozzi, A., \& Toth, P. (1981). Exact algorithms for the vehicle routing problem, based on spanning tree and shortest path relaxations. Mathematical Programming, 20(1), 255-282.

Christofides, N., \& Eilon, S. (1969). An algorithm for the vehicle-dispatching problem. Journal of the Operational Research Society, 20(3), 309-318.

Chen, A. L., Yang, G. K., \& Wu, Z. M. (2006). Hybrid discrete particle swarm optimization algorithm for capacitated vehicle routing problem. Journal of Zhejiang University-Science A, 7(4), 607-614.

Clarke, G., \& Wright, J. W. (1964). Scheduling of vehicles from a central depot to a number of delivery points. Operations Research, 12(4), 568-581.

Contardo, C., Cordeau, J. F., \& Gendron, B. (2014). A GRASP+ ILP-based metaheuristic for the capacitated location-routing problem. Journal of Heuristics, 20(1), 1-38.

Cornuejols, G., \& Harche, F. (1993). Polyhedral study of the capacitated vehicle routing problem. Mathematical Programming, 60(1-3), 21-52.

Dantzig, G. B., \& Ramser, J. H. (1959). The truck dispatching problem. Management Science, 6(1), 8091.

Duhamel, C., Lacomme, P., Prins, C., \& Prodhon, C. (2010). A GRASP $\times$ ELS approach for the capacitated location-routing problem. Computers \& Operations Research, 37(11), 1912-1923.

Escobar, J. W., Linfati, R., \& Toth, P. (2013). A two-phase hybrid heuristic algorithm for the capacitated location-routing problem. Computers \& Operations Research, 40(1), 70-79.

Escobar, J. W., Linfati, R., Toth, P., \& Baldoquin, M. G. (2014). A hybrid granular tabu search algorithm for the multi-depot vehicle routing problem. Journal of Heuristics, 20(5), 483-509.

Fourer, R., Gay, D. M., \& Kernighan, B. W. Ampl: a modeling language for mathematical programming. 2002. Duxbury Press.

Gendreau, M., Potvin, J. Y., Bräumlaysy, O., Hasle, G., \& Løkketangen, A. (2008). Metaheuristics for the vehicle routing problem and its extensions: A categorized bibliography. In The vehicle routing problem: latest advances and new challenges (pp. 143-169). Springer US.

Hemmelmayr, V. C., Cordeau, J. F., \& Crainic, T. G. (2012). An adaptive large neighborhood search heuristic for two-echelon vehicle routing problems arising in city logistics. Computers \& Operations Research, 39(12), 3215-3228.

ILOG, S. (2008). CPLEX optimization subroutine library guide and reference. System v11. 0 User's Guide.

Jansen, K. (1993). Bounds for the general capacitated routing problem. Networks, 23(3), 165-173.

Lavorato, M., Franco, J. F., Rider, M. J., \& Romero, R. (2012). Imposing radiality constraints in distribution system optimization problems. IEEE Transactions on Power Systems, 27(1), 172-180.

Letchford, A. N., \& Salazar-González, J. J. (2016). Stronger multi-commodity flow formulations of the (capacitated) sequential ordering problem. European Journal of Operational Research, 251(1), 7484.

Magnanti, T. L., Monma, C. L., \& Nemhauser, G. L. (1995). Network routing (Vol. 8). M. O. Ball (Ed.). Amsterdam: North-Holland.

Melechovský, J., Prins, C., \& Calvo, R. W. (2005). A metaheuristic to solve a location-routing problem with non-linear costs. Journal of Heuristics, 11(5-6), 375-391.

Miller, C. E., Tucker, A. W., \& Zemlin, R. A. (1960). Integer programming formulation of traveling salesman problems. Journal of the ACM (JACM), 7(4), 326-329.

Nagy, G., \& Salhi, S. (2007). Location-routing: Issues, models and methods. European Journal of Operational Research, 177(2), 649-672.

Prado, R. S., Silva, R. C. P., Neto, O. M., Guimarães, F. G., Sanches, D. S., London, J. B. A., \& Delbem, A. C. (2014). Differential evolution using ancestor tree for service restoration in power distribution systems. Applied Soft Computing, 23, 498-508.

Prodhon, C. (2011). A hybrid evolutionary algorithm for the periodic location-routing problem. European Journal of Operational Research, 210(2), 204-212. 
Prins, C. (2004). A simple and effective evolutionary algorithm for the vehicle routing problem. Computers \& Operations Research, 31(12), 1985-2002.

Prins, C., Prodhon, C., Ruiz, A., Soriano, P., \& Wolfler Calvo, R. (2007). Solving the capacitated location-routing problem by a cooperative Lagrangean relaxation-granular tabu search heuristic. Transportation Science, 41(4), 470-483.

Ralphs, T. K., Kopman, L., Pulleyblank, W. R., \& Trotter, L. E. (2003). On the capacitated vehicle routing problem. Mathematical Programming, 94(2), 343-359.

Salhi, S., \& Rand, G. K. (1989). The effect of ignoring routes when locating depots. European journal of operational research, 39(2), 150-156.

Ting, C. J., \& Chen, C. H. (2013). A multiple ant colony optimization algorithm for the capacitated location routing problem. International Journal of Production Economics, 141(1), 34-44.

Toth, P., \& Tramontani, A. (2008). An integer linear programming local search for capacitated vehicle routing problems. In The Vehicle Routing Problem: Latest Advances and New Challenges (pp. 275295). Springer US.

Toth, P., \& Vigo, D. (Eds.). (2014). Vehicle routing: problems, methods, and applications. Society for Industrial and Applied Mathematics.

Yu, B., Yang, Z. Z., \& Yao, B. (2009). An improved ant colony optimization for vehicle routing problem. European Journal of Operational Research, 196(1), 171-176.

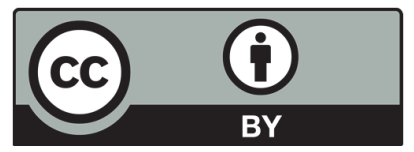

(C) 2017 by the authors; licensee Growing Science, Canada. This is an open access article distributed under the terms and conditions of the Creative Commons Attribution (CCBY) license (http://creativecommons.org/licenses/by/4.0/). 